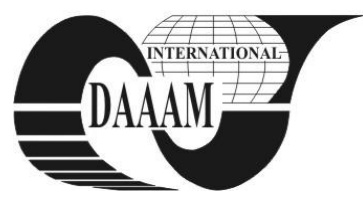

\title{
MATRIX EQUATION APPROACH FOR MIMO CONTROL DESIGN
}

\author{
VOLKOVA, N[atalia] \& PROKOP, R[oman]
}

\begin{abstract}
The contribution is focused on control design and simulation of MIMO linear continuous-time systems. Suitable and efficient tools for description and derivation are algebraic notions as rings, polynomial matrices, and Diophantine equations. Generalized MIMO PI controller design is studied for stable and unstable systems. Unified approach through matrix Diophantine equation can be applied in both cases. All stabilizing feedback controllers are obtained via solutions of matrix Diophantine equation. The methodology allows defining a scalar parameter for tuning and influencing of controller parameters. A Matlab, Simulink program implementation was developed for simulation and verification of the studied approach. Illustrative examples show the simplicity and flexibility of the proposed method for a simple two input - two output system.
\end{abstract}

Key words: MIMO system, diophantine equation, polynomial matrix, PI controller

\section{INTRODUCTION}

The analysis and control of MIMO systems is a very attractive and interesting research field. Methods and tools of SISO systems cannot be simply and trivially generalized into multivariable cases. The main problem relates to the noncommutativity of matrix multiplication. However, algebraic notions and tools can be successfully utilized also in the noncommutative case.

\section{SYSTEM DESCRIPTION}

A linear continuous-time multivariable (MIMO) system can be easily expressed by the Laplace transform technique in the form

$$
A(s) Y(s)=B(s) U(s)
$$

where $\tilde{A}(s), \widetilde{B}(s)$ are polynomial matrices. The matrix function can be then expressed by the left or right matrix fraction

$$
A(s)^{-1} B(s)=B_{R}(s) A_{R}(s)^{-1}
$$

\section{CONTROL DESIGN}

A basic (1 DOF) feedback control system is depicted in Fig. 1 .

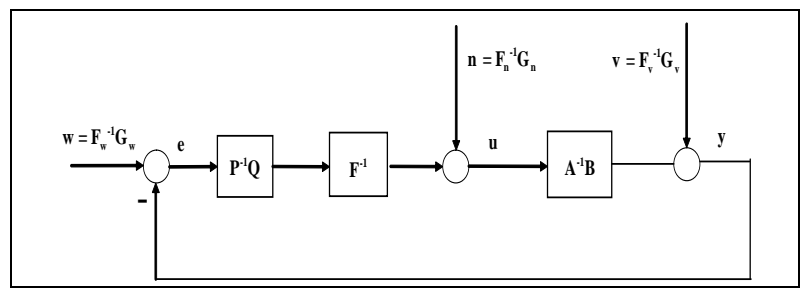

Fig. 1. One degree of freedom control system
Signals $w, n, v$ are reference, load disturbance and background noise, respectively. All are given by the left matrix fraction. A pre-compensator $F$ in Fig. 1 represents the conditions of divisibility. In the case of asymptotic tracking only, it is $F=F_{w}$. In the case of simultaneous asymptotic tracking and disturbance rejection and attenuation $F=F_{w} F_{v} F_{n}$.

The basic stability and asymptotic tracking in the sense of Fig. 1 is expressed by the solution of matrix Diophantine equation

$$
A(s) F(s) P_{R}(s)+B(s) Q_{R}(s)=M(s),
$$

where $\mathrm{M}(\mathrm{s})$ is a stable polynomial matrix with prescribed poles of its determinant. Resulting matrices $P_{R}, Q_{R}$ represent the right matrix fraction

$$
P(s)^{-1} Q(s)=Q_{R}(s) P_{R}(s)^{-1}
$$

The control law is then governed by the equation

$$
P(s)^{-1} F(s) U(s)=Q(s)(W(s)-Y(s)),
$$

which can be easily rewritten into differential equations. Now, it is necessary to propose the method for solution of matrix equation (2). For simpler cases, the solution can be found by means of elementary column operation, according to the scheme

$$
\left(\begin{array}{c|c}
A F & B \\
I & 0 \\
0 & I
\end{array}\right) \underset{\text { elementary column }}{\stackrel{\text { operations }}{\longrightarrow}}\left(\begin{array}{c|c}
M & 0 \\
P & Z_{1} \\
Q & Z_{2}
\end{array}\right)
$$

In more complex cases, the standard techniques based on Euclidean algorithms can be used, see Kučera, 1991, Vidyasagar, 1987.

\section{ILLUSTRATIVE EXAMPLES}

1) Stable system. A simple two input - two output (TITO) linear continuous-time system can express by the Laplace transform technique in the form

$$
\begin{aligned}
& y_{1}{ }^{\prime}(t)+2 y_{1}(t)+0,8 y_{2}(t)=5 u_{1}(t)+6 u_{2}(t) \\
& y_{2}{ }^{\prime}(t)+1,5 y_{2}(t)+0,6 y_{1}(t)=2 u_{1}(t)+3 u_{2}(t)
\end{aligned}
$$

The Laplace transform of equations (6) gives matrices $A, B$

$$
\left(\begin{array}{cc}
s+a_{1} & a_{2} \\
a_{3} & s+a_{4}
\end{array}\right)\left(\begin{array}{l}
Y_{1}(s) \\
Y_{2}(s)
\end{array}\right)=\left(\begin{array}{ll}
b_{1} & b_{2} \\
b_{3} & b_{4}
\end{array}\right)\left(\begin{array}{l}
U_{1}(s) \\
U_{2}(s)
\end{array}\right)
$$


The stabilization matrix Diophantine equation (3) takes the form

$$
\begin{aligned}
& \left(\begin{array}{cc}
s+a_{1} & a_{2} \\
a_{3} & s+a_{4}
\end{array}\right)\left(\begin{array}{ll}
s & 0 \\
0 & s
\end{array}\right)\left(\begin{array}{ll}
p_{1} & p_{2} \\
p_{3} & p_{4}
\end{array}\right)+\left(\begin{array}{ll}
b_{1} & b_{2} \\
b_{3} & b_{4}
\end{array}\right) \\
& \left(\begin{array}{cc}
q_{1} s+q_{0} & q_{5} s+q_{4} \\
q_{3} s+q_{2} & q_{7} s+q_{6}
\end{array}\right)=\left(\begin{array}{cc}
\left(s+m_{0}\right)^{2} & 0 \\
0 & \left(s+m_{0}\right)^{2}
\end{array}\right)
\end{aligned}
$$

Then the scheme (5) can be applied and the result is in the form of generalized PI controller:

$$
\begin{aligned}
& u_{1}=q_{1} e_{1}+q_{0} \int e_{1}(\tau) d \tau+q_{5} e_{2}+q_{4} \int e_{2}(\tau) d \tau \\
& u_{2}=q_{3} e_{1}+q_{2} \int e_{1}(\tau) d \tau+q_{7} e_{2}+q_{6} \int e_{2}(\tau) d \tau
\end{aligned}
$$

where controller parameters were obtained by elementary column operations according scheme (5) in the form

$$
\begin{aligned}
q_{1} & =2 m_{0}-0,8 & q_{5} & =-4 m_{0}+2,2 \\
q_{0} & =m_{0}^{2} & q_{4} & =-2 m_{0}^{2} \\
q_{3} & =-\frac{4}{3} m_{0}+\frac{1}{3} & q_{7} & =\frac{10}{3} m_{0}-\frac{5,9}{3} \\
q_{2} & =-\frac{2}{3} m_{0}^{2} & q_{2} & =\frac{5}{3} m_{0}^{2}
\end{aligned}
$$

In (9) $e_{i}$ are naturally tracking errors and $m_{0}>0$ is a tuning parameter influencing control behavior.

2) Unstable system. A two input two output (TITO) linear continuous-time system can be expressed in the form

$$
\begin{gathered}
y_{1}{ }^{\prime}(t)+y_{1}(t)+y_{2}(t)=u_{1}(t)+0,5 u_{2}(t) \\
y_{2}{ }^{\prime}(t)+0,5 y_{2}(t)+2 y_{1}(t)=0,8 u_{1}(t)+2 u_{2}(t) \\
\left(\begin{array}{ll}
s+1 ; & 1 \\
2 ; & s+0.5
\end{array}\right)\left(\begin{array}{l}
Y_{1}(s) \\
Y_{2}(s)
\end{array}\right)=\left(\begin{array}{rr}
1 ; & 0.5 \\
0.8 ; & 2
\end{array}\right)\left(\begin{array}{l}
U_{1}(s) \\
U_{2}(s)
\end{array}\right) \\
q_{1}=2,5 m_{0}-0,65 \quad q_{5}=-0,6 m_{0}-1,1 \\
q_{0}=1,25 m_{0}^{2} \\
q_{3}=-m_{0}-0,75 \\
q_{2}=-0,5 m_{0}^{2}
\end{gathered} \quad \begin{aligned}
& q_{7}=-0,3 m_{0}^{2} \\
& q_{2}=0,25 m_{0}+0,19
\end{aligned}
$$

\section{SIMULATION RESULTS}

Suitable environment for modeling and simulation of dynamic systems is Matlab and Simulink. The Simulink scheme for two input - two output stable system (7) with controller (10) is depicted in Fig. 2.

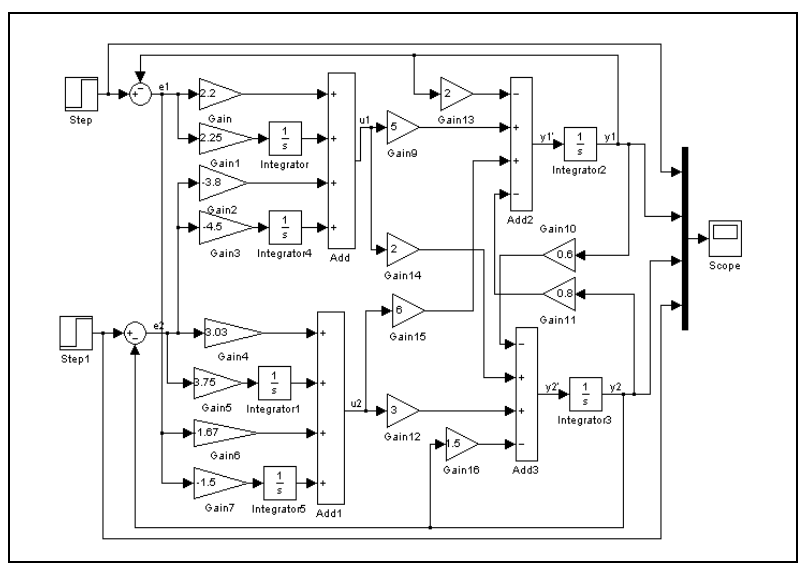

Fig. 2. Simulink scheme of feedback stable system
Control responses of stable TITO system (example 1) for tuning parameter $\mathrm{m}_{0}=1,5$ and $\mathrm{m}_{0}=3$ are performed in Fig. 3 . Representation of unstable TITO system (example 2) for tuning parameter $\mathrm{m}_{0}=2$ and $\mathrm{m}_{0}=3$ is shown in Fig. 4 .

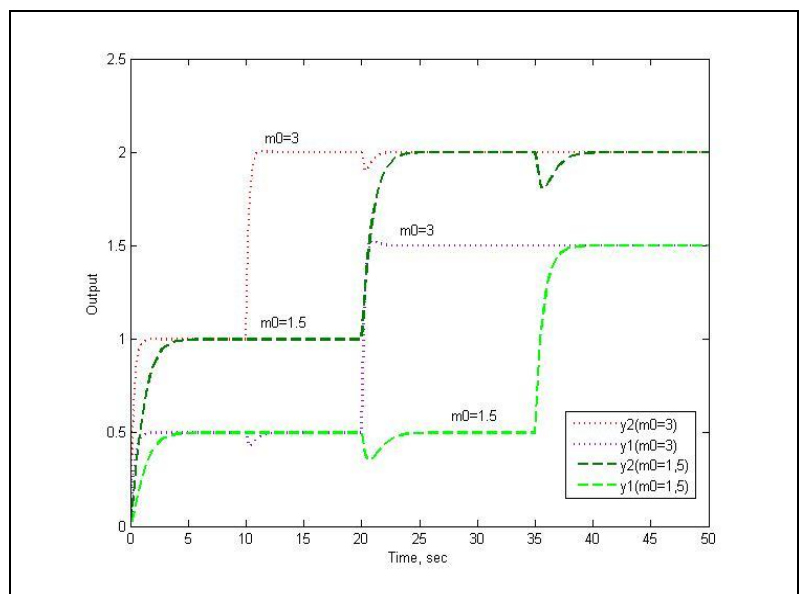

Fig. 3. Control responses for $\mathrm{m}_{0}=1,5$ and $\mathrm{m}_{0}=3$

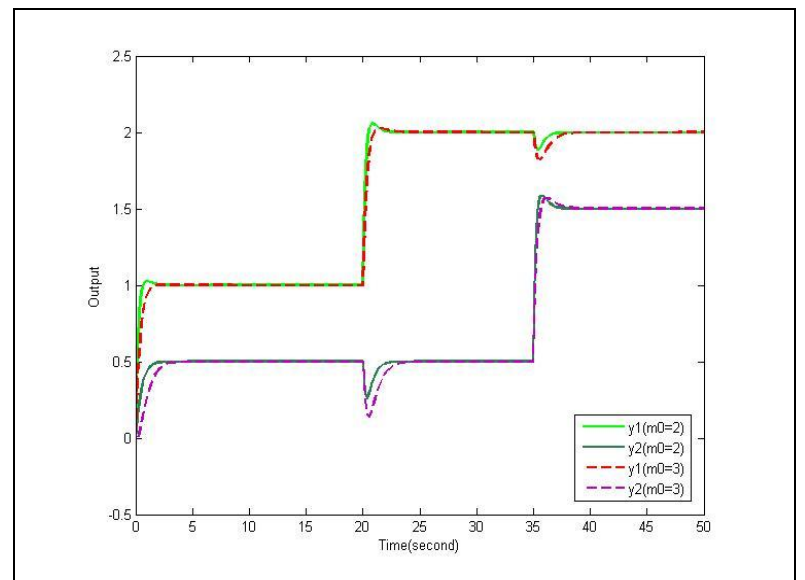

Fig. 4. Control responses for $\mathrm{m}_{0}=2$ and $\mathrm{m}_{0}=3$

\section{CONCLUSION}

The paper deals with multivariable control of continuoustime linear systems. The controller design is performed through applying matrix Diophantine equation. This approach enables to define one or a couple scalar tuning parameters for influencing of control behavior. All simulations and satisfactory results are clearly demonstrated in the Matlab, Simulink environment.

\section{REFERENCES}

Åström, K.J. and T. Hägglund (1995). PID Controllers: Theory, Design and Tuning, Research Triangle Park, NC: Instrumental Society of America

Kučera, V. (1991). Analysis and Design of Discrete Linear Control systems, Prentice Hall, London - A survey," Automatica, Vol. 29, pp. 1361-75

Kučera, V. (1993). Diophantine equations in control - A survey, Automatica, Vol. 29, pp. 1361-75

Kaczorek, T. (1985). Two-dimensional Linear Systems, Springer-Verlag, Berlin

Prokop, R. and J.P. Corriou (1997). Design and analysis of simple robust controllers, Int. J. Control, Vol. 66, pp. 905921

Rosenwasser, E.N. and B.P. Lampe (2006). Multivariable Computer-controlled Systems, Springer-verlag, Berlin

Vidyasagar, M. (1987). Control system synthesis: a factorization approach, MIT Press, Cambridge, M.A. 